\title{
Correction to: A Novel Methodology Using Dexamethasone to Induce Neuronal Differentiation in the CNS-Derived Catecholaminergic CAD Cells
}

\author{
Ekkaphot Khongkla $^{1} \cdot$ Kwanchanok Uppakara $^{2} \cdot$ Nittaya Boonmuen $^{1} \cdot$ Kanit Bhukhai $^{1} \cdot$ Witchuda Saengsawang $^{1,3,4}$
}

Published online: 16 June 2021

(c) Springer Science+Business Media, LLC, part of Springer Nature 2021

\section{Correction to: Cellular and Molecular Neurobiology https://doi.org/10.1007/s10571-021-01109-z}

The original version of this article unfortunately contained a typo in corresponding author name.

The corresponding author name is Witchuda Saengsawang instead it was published incorrectly as Witchuda Seangsawang.

The original article has been corrected.

Publisher's Note Springer Nature remains neutral with regard to jurisdictional claims in published maps and institutional affiliations.

The original article can be found online at https://doi.org/10.1007/ s10571-021-01109-z.

Witchuda Saengsawang

witchudasaeng@gmail.com

1 Department of Physiology, Faculty of Science, Mahidol University, 272 Rama 6 Rd. Payathai, Ratchathewi district, Bangkok 10400, Thailand

2 Toxicology Graduate Program, Faculty of Sciene, Mahidol University, Bangkok, Thailand

3 Faculty of Science, Center of Neuroscience, Mahidol University, Bangkok, Thailand

4 Faculty of Science, Excellent Center for Drug Discovery (ECDD), Mahidol University, Bangkok, Thailand 